\title{
AVALIAÇÃO DE PERDAS INVISÍVEIS NA COLHEITA MECANIZADA EM DOIS FLUXOS DE MASSA DE CANA-DE-AÇÚCAR
}

\author{
JORGE L. M. NEVES ${ }^{1}$, PAULO S. G. MAGALHÃES ${ }^{2}$, EDSON E. MORAES ${ }^{3}$, \\ FAUSTO V. M. ARAÚJO ${ }^{4}$
}

\begin{abstract}
RESUMO: Neste trabalho, analisou-se o comportamento da colhedora de cana-de-açúcar picada frente a dois fluxos de alimentação de matéria-prima, mensurando-se as perdas invisíveis, impossíveis de serem quantificadas diretamente no campo, a eficiência de limpeza da colhedora e a velocidade do ar na saída dos extratores. Os ensaios foram realizados sob condições controladas no Centro de Tecnologia Copersucar, em janeiro de 2000. Os resultados apontaram que o fluxo de massa de cana que alimenta a colhedora, na faixa avaliada, não tem influência nas magnitudes das perdas invisíveis totais de cana e na eficiência de limpeza da matéria-prima. A velocidade média do ar na saída do extrator primário foi de $12,0 \mathrm{~m} \mathrm{~s}^{-1}$, e no extrator secundário, de $9,2 \mathrm{~m} \mathrm{~s}^{-1}$, com coeficiente de variação médio de $21 \%$, indicando que o baixo desempenho de limpeza da colhedora pode estar associado às diferenças de velocidade do ar nos extratores. Por meio da análise dos dados quantificados nos ensaios, concluiu-se que as perdas invisíveis no processamento da cana na colhedora foram de $10 \%$, e a eficiência de limpeza, de $87 \%$.
\end{abstract}

PALAVRAS-CHAVE: colhedora de cana picada, perdas na colheita, fluxo de ar.

\section{EVALUATION OF INVISIBLE LOSSES IN MECHANICAL HARVESTING IN TWO SUGAR CANE MASS FLOWS}

\begin{abstract}
In this work the performance of a sugar cane chopped harvester was analysed when fed with two sugar cane mass flows, measuring the invisible losses, which are impossible to measure in the field, harvester sugar cane cleaning efficiency and air velocity on extractors exit. The trial was done under controlled conditions at Copersucar Technology Center in January 2000. The results showed that the flow of sugar cane through the harvester doesn't influence the magnitudes of total invisible losses and raw material cleaning efficiency. The mean air velocity on the primary extractors exit was $12.0 \mathrm{~m} \mathrm{~s}^{-1}$, and $9.2 \mathrm{~m} \mathrm{~s}^{-1}$ on the secondary extractor, with a coefficient of variation of $21 \%$, indicating that the poor cleaning performance of the harvester could be related to air velocity difference inside the extractor. Analyzing the data collected in the trials, it was possible to conclude that invisible losses in sugar cane harvester were $10 \%$ and the cleaning efficiency was $87 \%$.
\end{abstract}

KEYWORDS: sugar cane harvester, harvesting losses, air flow.

\section{INTRODUÇÃO}

O Brasil é o maior produtor e também o país onde se produz álcool e açúcar a menor custo. Seja pelo baixo custo, seja pelos bons indicadores de sustentabilidade energética, ambiental e social, o etanol brasileiro vem consolidando seu espaço no mercado interno e internacional.

Atualmente, estão sendo implantadas 50 novas usinas de açúcar e etanol no País, projetando o crescimento da produção de cana de mais de $50 \%$ nos próximos oito anos. De forma muito simplificada, pode-se dizer que a competitividade brasileira deve-se à capacidade de produzir matéria-prima de baixo custo e também à infra-estrutura e capacidade gerencial, sobretudo, existentes na região Centro-Sul do País.

\footnotetext{
${ }^{1}$ Eng $^{\mathrm{o}}$ Mecânico, Doutor em Engenharia Agrícola, Pesquisador do CTCanavieira, Piracicaba - SP.

${ }^{2}$ Professor Titular, FEAGRI/UNICAMP, Pesquisador do NIPE/UNICAMP, Campinas - SP, graziano@ agr.unicamp.br

${ }^{3}$ Eng $^{\mathrm{o}}$ Agrícola, M.Sc., Pesquisador do CTCanavieira, Piracicaba - SP.

${ }^{4}$ Aluno de Engenharia Agrícola, FEAGRI, estagiário do CTC, Piracicaba - SP.

Recebido pelo Conselho Editorial em: 20-6-2006
}

Aprovado pelo Conselho Editorial em: 19-10-2006 
No âmbito da produção agrícola, é importante que se reconheça que $60 \%$ do custo de produção do etanol e açúcar é representado pela matéria-prima, sendo o restante representado pelo processamento e custos administrativos, de transporte e de distribuição. Nesse âmbito, a colheita mecanizada da cana, que tem crescido nos últimos anos, é tema importante e altamente relevante. Atualmente, $40 \%$ da área colhida de cana-de-açúcar utiliza o processo mecanizado de colheita, com ou sem queima prévia para limpeza do canavial, principalmente em áreas com topografia adequada e com problemas relacionados à disponibilidade de mão-de-obra. Conforme os dados dos próprios fabricantes, a maioria das colhedoras de cana picada em operação no País é das marcas Case (CNH-New Holland) e Cameco (John Deere), que trabalham no campo segundo o mesmo princípio de operação e utilizam os mesmos componentes e sistemas de processamento de cana. Contudo, a adoção do sistema mecanizado de colheita de cana picada introduz certos inconvenientes, tais como aumento dos índices de impurezas na carga, que implicam a redução da qualidade tecnológica da matéria-prima fornecida para moagem e perdas de cana no campo (MAGALHÃES \& BRAUNBECK, 1998).

Segundo MORAES (1992), a cana colhida por colhedoras de cana picada apresenta índices de impurezas 2,7 vezes maiores que a cana cortada manualmente e carregada mecanicamente. KROES \& HARRIS (1996) destacam que a utilização do corte mecânico pode causar perdas de cana e de caldo e danos na base da soqueira, que reduzem consideravelmente a brotação e proporcionam o ataque de doenças e pragas, refletindo na perda de produtividade da safra subseqüente.

NEVES et al. (2004), realizando ensaios com monitor de perdas durante a colheita, relatam que as perdas visíveis no campo foram da ordem de 5\%, verificando-se a correspondência do aumento das perdas de pedaços de cana com o acréscimo da rotação do extrator primário. Também informam que, em todos os ensaios, a incidência de impurezas na carga diminuiu, a massa foliar no campo aumentou e a carga transportada foi maior com o aumento da rotação do extrator primário.

RIPOLI et al. (2001a), realizando testes de campo com duas colhedoras Claas, modelos Ventor e CC3000, processando a variedade de cana-de-açúcar RB 83-5089, sem queimar, em área com produtividade média de $126 \mathrm{Mg} \mathrm{ha}^{-1}$, e operando com quatro velocidades de translação $(0,47$; 0,$92 ; 1,50$ e $2,08 \mathrm{~m} \mathrm{~s}^{-1}$ ), concluíram que, além do custo da tonelada de cana colhida diminuir, as capacidades efetiva e operacional de campo aumentaram com o aumento da velocidade das máquinas, assim como as perdas de cana e o índice de impureza vegetal não aumentaram com as colhedoras trabalhando em velocidades maiores. RIPOLI et al. (2001b) chegaram às mesmas conclusões operando uma colhedora Brastoft A 7700, trabalhando em outras quatro velocidades de translação $\left(0,36 ; 0,75 ; 1,47\right.$ e $\left.2,14 \mathrm{~m} \mathrm{~s}^{1}\right)$, processando a mesma variedade de cana, sem queimar, em área com 14,5 $\mathrm{Mg} \mathrm{ha}^{-1}$ de produtividade. NEVES et al. (2003) quantificaram as perdas invisíveis que ocorrem durante a colheita de cana-de-açúcar no conjunto do sistema: rolos transportadores; rolos síncronos; ventiladores e elevador, e no sistema de corte de base. Os resultados mostram que as perdas invisíveis dependem da variedade da cana e do estado das facas do cortador de base. Essas perdas foram significativas e da ordem de 2,1 a 5,4\%. Os autores utilizaram, nesse ensaio, cana-de-açúcar sem palha e mantiveram, em todos os testes, fluxo constante de cana.

O objetivo deste trabalho foi realizar avaliação do desempenho da colhedora de cana-deaçúcar submetida à taxa de alimentação variável de cana crua e com folhas, obtendo os dados das perdas invisíveis, impossíveis de serem quantificadas diretamente no campo, das eficiências de limpeza efetuadas pelos extratores da colhedora e a velocidade do fluxo de ar na saída dos extratores.

\section{MATERIAL E MÉTODOS}

O ensaio foi realizado na oficina mecânica de protótipos agrícolas do Centro de Tecnologia Copersucar (CTC), localizado em Piracicaba - SP, em janeiro de 2000. Foram selecionadas duas variedades de cana, pretendendo-se avaliar, comparativamente, produtos que se caracterizam pela 
diferença no teor de fibra (Tabela 1), sendo classificadas de acordo com os critérios estabelecidos na Seção de Tecnologia de Ensaio de Competição (CTFT-4) do CTC, que estabelece que canas com alto teor de fibra apresentam de 12,5 a 14,0\% de fibra, com teores entre 11,0 e 12,5\% são considerados médios teores, e entre 9,5 e $11,0 \%$, baixos teores.

TABELA 1. Caracterização das variedades de cana-de-açúcar quanto ao teor de fibra e estado em relação ao porte do canavial.

\begin{tabular}{cccc}
\hline Variedade & Estado & Fibra (\%) & Classificação quanto ao teor de fibra \\
\hline RB72454 & Ereta & 12,17 & médio \\
SP80-1842 & Ereta & 13,27 & alto \\
\hline
\end{tabular}

A colhedora foi alimentada com amostras de cana-de-açúcar com palha por meio de uma esteira transportadora com $2 \mathrm{~m}$ de comprimento, acionada por motor hidráulico orbital de deslocamento volumétrico constante, acionado pela bomba hidráulica da colhedora. Manteve-se a velocidade da esteira constante em aproximadamente $1,7 \mathrm{~m} \mathrm{~s}^{-1}$

Cada amostra foi constituída de um feixe de 20 ou 30 canas com folhas, pesada antes de cada repetição e distribuída ao longo da esteira com espaçamento entre canas semelhante ao observado no campo, onde geralmente se encontram 10 a 15 canas por metro, num total de dez repetições em cada ensaio, simulando, dessa maneira, dois diferentes fluxos de cana para cada variedade.

O experimento foi delineado para ser realizado em blocos ao acaso, com repetições. Quando não houve interação entre os blocos, fez-se a comparação entre as médias dos diferentes tratamentos em cada bloco, fornecendo a diferença mínima significante (dms) entre as médias, pelo teste de Tukey.

A análise de variância das médias foi realizada considerando como blocos as duas variedades e como tratamentos os dois fluxos de massa de cana processados pela colhedora. Foram avaliadas as perdas invisíveis e a eficiência de limpeza da matéria-prima.

Para a realização deste ensaio, foi utilizada uma colhedora Cameco CH 2500, modelo 1998, com potência de $224 \mathrm{~kW}$, de esteiras e um transbordo para recolhimento dos rebolos. Para a coleta do material expelido pelos extratores primário e secundário, os capuzes desses componentes foram equipados com telas de náilon tipo mosquiteiros (Figura 1).

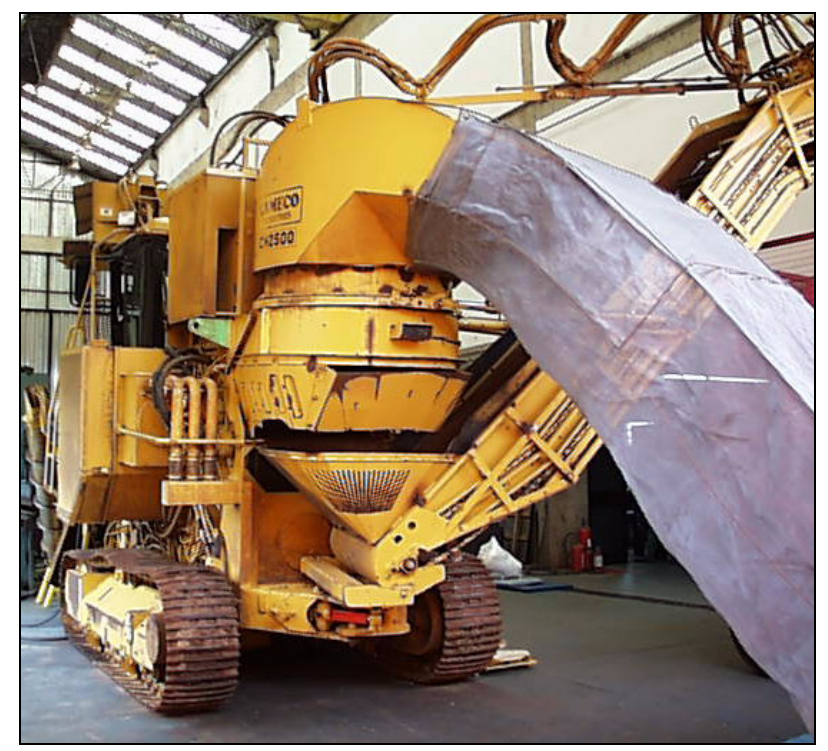

FIGURA 1. Área do ensaio - colhedora ensaiada com detalhe da tela de náilon instalada no capuz do extrator primário.

Durantes os ensaios, a velocidade do ventilador do extrator primário foi mantida em $22 \mathrm{rps}$, e a do ventilador do extrator secundário, em 28 rps. 
Para o levantamento de dados técnicos de funcionamento da colhedora, determinou-se preliminarmente a velocidade de saída do ar dos extratores primário e secundário. A área de abertura das carenagens dos extratores foi dividida em subáreas, utilizando-se de arames de aço (Figura 2), e as leituras de velocidade foram tomadas nos centros dessas subáreas resultantes, utilizando-se de anemômetro digital de leitura direta com faixa de leitura de 0 a $45 \mathrm{~m} \mathrm{~s}^{-1}$.

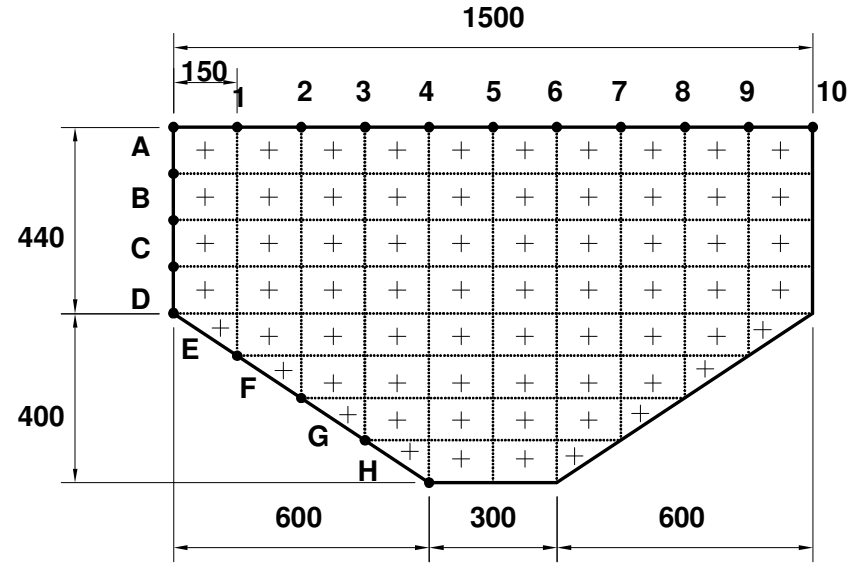

(a)

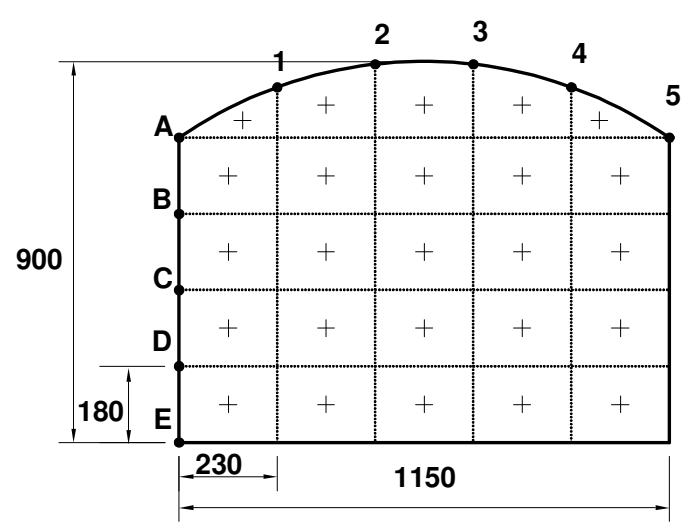

(b)

FIGURA 2. Abertura do extrator primário (a) e do extrator secundário (b) (medidas em mm).

Foram utilizados dois fluxos de massa de cana que alimentam a máquina, calculados da seguinte maneira:

$$
\mathrm{Q}=(\mathrm{N})\left(\frac{1}{\mathrm{~L}}\right) \mathrm{v}
$$

em que,

$\mathrm{Q}$ - fluxo de cana-de-açúcar alimentando a colhedora, $\mathrm{Mg} \mathrm{h}^{-1}$;

$\mathrm{N}$ - massa do feixe de cana-de-açúcar da amostra com feixes de 20 ou 30 canas, $\mathrm{kg}$;

L - comprimento útil da esteira de alimentação de cana na máquina, $\mathrm{m}$, e

$v$ - velocidade da esteira, $\mathrm{km} \mathrm{h}^{-1}$.

As perdas invisíveis da colhedora foram levantadas em todos os conjuntos da máquina, exceto no corte basal. Além das perdas invisíveis, foram obtidos os dados de eficiência de limpeza $(\mathrm{EF} \%)$ na base seca e úmida.

As perdas invisíveis (PI\%) foram determinadas, utilizando-se da eq. (2), e a eficiência de limpeza, pela eq. (3).

$$
\begin{aligned}
& \mathrm{PI} \%=\frac{\mathrm{PSA}-(\mathrm{TLC}+\mathrm{TLP}+\mathrm{TLM}+\mathrm{PPC}+\mathrm{PPP}+\mathrm{PPM})}{\mathrm{PSA}} 100 \\
& \mathrm{EF} \%=\left(1-\frac{\mathrm{PPC}}{\mathrm{PPC}-\mathrm{MFC}}\right) 100 \\
& \mathrm{MFC}=\mathrm{PPP}+\mathrm{PPM}
\end{aligned}
$$

em que,

MFC - massa foliar recolhida da máquina e do chão, g;

PPC - massa da palha e palmito coletados na carroceria, $\mathrm{g}$;

PPM - massa da palha e palmito coletados na máquina, g;

PPP - massa da palha e palmito coletados no chão, g;

PSA - massa total das amostras de canas, g;

TLC - massa dos rebolos coletados na carroceria, g;

TLM - massa dos rebolos e pedaços coletados na máquina, g, e

TLP - massa dos rebolos e pedaços coletados no chão, g. 
Por massa foliar, entende-se a quantidade de palha e palmito coletados na máquina e no chão, mais a palha e palmito coletados nas telas montadas nas saídas de ar da carenagem dos extratores primário e secundário.

\section{RESULTADOS E DISCUSSÃO}

A velocidade média do ar na saída do extrator primário da colhedora foi de $11,6 \mathrm{~m} \mathrm{~s}^{-1}$ com tela, e de 12,0 $\mathrm{m} \mathrm{s}^{-1}$ sem tela, e do extrator secundário foi de $8,6 \mathrm{~m} \mathrm{~s}^{-1}$ e 9,2 $\mathrm{m} \mathrm{s}^{-1}$ com e sem tela, respectivamente. Nas Tabelas 2 e 3, apresentam-se os dados de velocidade média do extrator primário, sem tela, nos pontos coletados com o elevador posicionado do lado direito e esquerdo, respectivamente.

TABELA 2. Velocidade média de saída de ar do extrator primário, sem tela, nos pontos coletados com o elevador posicionado do lado direito.

\begin{tabular}{crrrrrrrrrrrr}
\hline \multirow{2}{*}{ Posição } & 1 & 2 & 3 & 4 & 5 & 6 & 7 & 8 & 9 & 10 & Média & C.V. \\
\cline { 2 - 11 } & & \multicolumn{7}{c}{ Velocidade } & $\left(\mathrm{m} \mathrm{s}^{-1}\right)$ \\
A & 20,3 & 20,2 & 21,3 & 20,2 & 20,2 & 20,3 & 19,5 & 17,1 & 13,5 & 13,2 & 18,6 & 15,9 \\
B & 18,7 & 17,6 & 18,7 & 18,2 & 16,6 & 17,2 & 18,5 & 16,1 & 10,4 & 9,0 & 16,1 & 21,7 \\
C & 17,4 & 15,7 & 16,4 & 16,8 & 15,8 & 16,2 & 17,5 & 16,0 & 8,3 & 6,6 & 14,7 & 26,4 \\
D & 16,6 & 15,0 & 14,9 & 16,1 & 15,7 & 14,9 & 15,2 & 13,4 & 9,8 & 9,4 & 14,1 & 17,9 \\
E & & 13,6 & 13,1 & 14,6 & 15,4 & 13,1 & 13,1 & 11,1 & 9,3 & & 12,9 & 14,9 \\
F & & & 14,3 & 15,1 & 11,6 & 10,4 & 9,5 & 9,9 & & & 11,8 & 20,1 \\
G & & & 15,7 & 16,3 & 13,2 & 7,6 & 6,6 & 8,6 & & & 11,3 & 37,6 \\
H & & & & 14,9 & 8,7 & & & & & & 11,8 & 37,2 \\
\hline Média & 18,3 & 16,4 & 16,3 & 16,5 & 14,7 & 14,3 & 14,3 & 13,2 & 10,3 & 9,5 & 13,8 & \\
C.V. (\%) & 8,7 & 14,7 & 16,5 & 11,0 & 22,9 & 28,9 & 32,2 & 24,9 & 19,2 & 27,7 & & \\
\hline C.V.
\end{tabular}

C.V. - coeficiente de variação.

TABELA 3. Velocidade média de saída de ar do extrator primário, sem tela, nos pontos coletados com o elevador posicionado do lado esquerdo.

\begin{tabular}{|c|c|c|c|c|c|c|c|c|c|c|c|c|}
\hline \multirow{2}{*}{ Posição } & 1 & 2 & 3 & 4 & 5 & 6 & 7 & 8 & 9 & 10 & Média & \multirow{2}{*}{$\begin{array}{l}\text { C.V. } \\
(\%)\end{array}$} \\
\hline & \multicolumn{11}{|c|}{ Velocidade $\left(\mathrm{m} \mathrm{s}^{-1}\right)$} & \\
\hline A & 14,7 & 14,7 & 15,6 & 14,5 & 14,5 & 14,3 & 13,6 & 11,9 & 9 & 8,9 & 13,2 & 18,4 \\
\hline B & 13,6 & 11,9 & 12,3 & 12,8 & 12,3 & 13 & 12,6 & 10,4 & 4,2 & 3,4 & 10,7 & 34,8 \\
\hline $\mathrm{C}$ & 11,5 & 10,7 & 11 & 11,4 & 11,8 & 11,8 & 11,2 & 9,2 & 4,6 & 3,5 & 9,7 & 31,7 \\
\hline D & 13 & 10,9 & 10,5 & 11 & 11 & 10,7 & 10,7 & 8,7 & 5,4 & 5,3 & 9,7 & 25,9 \\
\hline $\mathrm{E}$ & & 12,5 & 11,2 & 11,2 & 11 & 10,5 & 9,6 & 8,4 & 6,1 & & 10,1 & 20,0 \\
\hline $\mathrm{F}$ & & & 10,7 & 12,6 & 11,6 & 8,1 & 8,3 & 8,1 & & & 9,9 & 20,1 \\
\hline $\mathrm{G}$ & & & 12 & 12,8 & 11 & 4,8 & 4,3 & 6,4 & & & 8,6 & 44,6 \\
\hline $\mathrm{H}$ & & & & 11,9 & & & & & & & 11,9 & \\
\hline médias & 13,2 & 12,2 & 11,9 & 12,3 & 11,9 & 10,5 & 10,1 & 9,0 & 5,9 & 5,3 & 10,23 & \\
\hline C.V. (\%) & 10,1 & 13,2 & 14,8 & 9,4 & 10,5 & 30,3 & 30,6 & 19,5 & 32,2 & 48,5 & & \\
\hline
\end{tabular}

C.V. - coeficiente de variação.

Pelos dados obtidos de velocidades de saída do ar no extrator primário, observa-se variação com aumento de suas magnitudes da direita para a esquerda (posição 1 para 10) e diminuição da parte superior para a inferior (posição A para H), Figura 2. Essa variação é conseqüência do sentido horário de giro das hélices do ventilador, do formato das aberturas para entrada de ar no bojo e da carenagem do anel e capuz do extrator primário direcionar a saída de ar para a parte superior esquerda de sua abertura. Como decorrência disso, tem-se o lançamento de pedaços estilhaçados de cana quando se aumenta a rotação do ventilador ou, com a sua diminuição, a ineficiência da 
limpeza da matéria-prima com excesso de palha na carga de cana a ser transportada para a usina. A velocidade média de saída do ar do extrator primário foi superior quando o elevador foi posicionado do lado direito da colhedora, mas, em ambas as posições, a distribuição foi equivalente, com coeficiente de variação médio de $21 \%$.

Observa-se que as velocidades de saída do ar nos extratores foram inferiores aos valores obtidos por MORAES (1992), trabalhando com a colhedora Dedini DM6000, que registrou $22,3 \mathrm{~m} \mathrm{~s}^{-1}$ no extrator primário e $20,9 \mathrm{~m} \mathrm{~s}^{-1}$ no extrator secundário.

Os resultados obtidos neste trabalho apresentam velocidades mais próximas das indicadas por DICK \& RIDGE (1989), que recomendam utilizar velocidade de até $16 \mathrm{~m} \mathrm{~s}^{-1}$, para um valor ótimo de operação no extrator primário, e evitar velocidades acima de $20 \mathrm{~m} \mathrm{~s}^{-1}$ para não aumentar muito as perdas de cana.

O resultado das perdas invisíveis totais da colhedora, exceto o conjunto de corte de base, e a eficiência de limpeza do extrator primário estão apresentados na Tabela 4.

TABELA 4. Levantamento de perdas invisíveis e eficiência de limpeza, para a colhedora Cameco CH 2500, utilizando facas dos rolos picadores síncronos novos, sem computar as perdas devido ao corte de base.

\begin{tabular}{ccccccc}
\hline Variedade & $\begin{array}{c}\text { Fluxo de cana } \\
\left(\mathrm{Mg} \mathrm{h}^{-1}\right)\end{array}$ & $\begin{array}{c}\text { Perdas } \\
\text { Invisíveis } \\
\text { Totais }(\%)\end{array}$ & $\begin{array}{c}\text { Perdas de } \\
\text { Lascas } \\
(\%)\end{array}$ & $\begin{array}{c}\text { Perdas de } \\
\text { Serragem }\end{array}$ & \multicolumn{2}{c}{$\begin{array}{c}\text { Eficiência de Limpeza } \\
(\%)\end{array}$} \\
\hline \multirow{2}{*}{ RB72454 } & 137,5 & $10,3 \mathrm{a}$ & 8,1 & 2,2 & $80,5 \mathrm{a}$ & $87,1 \mathrm{a}$ \\
& 205,2 & $10,7 \mathrm{a}$ & 9,2 & 1,55 & $82,5 \mathrm{a}$ & $87,0 \mathrm{a}$ \\
\hline \multirow{2}{*}{ SP80-1842 } & 110,8 & $9,8 \mathrm{a}$ & 7,1 & 2,73 & $78,3 \mathrm{a}$ & $87,4 \mathrm{a}$ \\
& 160,5 & $9,9 \mathrm{a}$ & 8,1 & 1,84 & $82,9 \mathrm{a}$ & $89,5 \mathrm{a}$ \\
\hline
\end{tabular}

Médias seguidas por letras distintas diferem entre si, a 5\% de significância, pelo teste de Tukey.

Observa-se, na Tabela 4, que as médias não diferem a 5\% de probabilidade $($ Tukey, dms $=$ $1,067)$.

As perdas invisíveis totais não dependeram da variedade de cana, o que pode ser justificado pela proximidade do teor de fibra (\%) verificado nas duas variedades ensaiadas (Tabela 1). Em relação aos tratamentos com fluxos de cana com $45 \%$ de diferença, as perdas invisíveis totais não apresentaram diferenças significativas a $5 \%$ de probabilidade, com variação de $0,1 \%$ na variedade SP80-1842 e 0,4\% na variedade RB72454, sendo o total de perdas invisíveis de 9,8 a 10,7\%, respectivamente, sem incluir as perdas referentes ao conjunto corte basal.

$\mathrm{O}$ fato de as perdas invisíveis serem iguais quando se processam fluxos de massa de cana-deaçúcar diferentes, confirma os resultados a que chegaram RIPOLI et al. (2001a e 2001b) que, quando trabalharam com colhedoras operando no campo a velocidades de translação diferentes, obtiveram o mesmo valor de perdas de cana-de-açúcar.

As perdas invisíveis levantadas nos ensaios, quando em serragem e lascas (Figura 3), separadas das impurezas vegetais lançadas pelo extrator primário, são diferentes para cada fluxo de cana, sendo que as perdas invisíveis na forma de lascas aumentam com o aumento do fluxo de cana processado pela colhedora, e as de serragem diminuem, mantendo-se constante as perdas totais.

A análise química desse material mostrou, ainda, que as perdas invisíveis apresentavam grande quantidade de açúcar. Para a variedade SP80-1842, que estava com 14,1\% de pol, as lascas estavam com $13,0 \%$, e a serragem, com $13,9 \%$ de pol.

A eficiência de limpeza foi a mesma em relação às duas variedades analisadas nos dois tratamentos, devido à quantidade de massa foliar das duas variedades serem praticamente as mesmas nesse ensaio. 


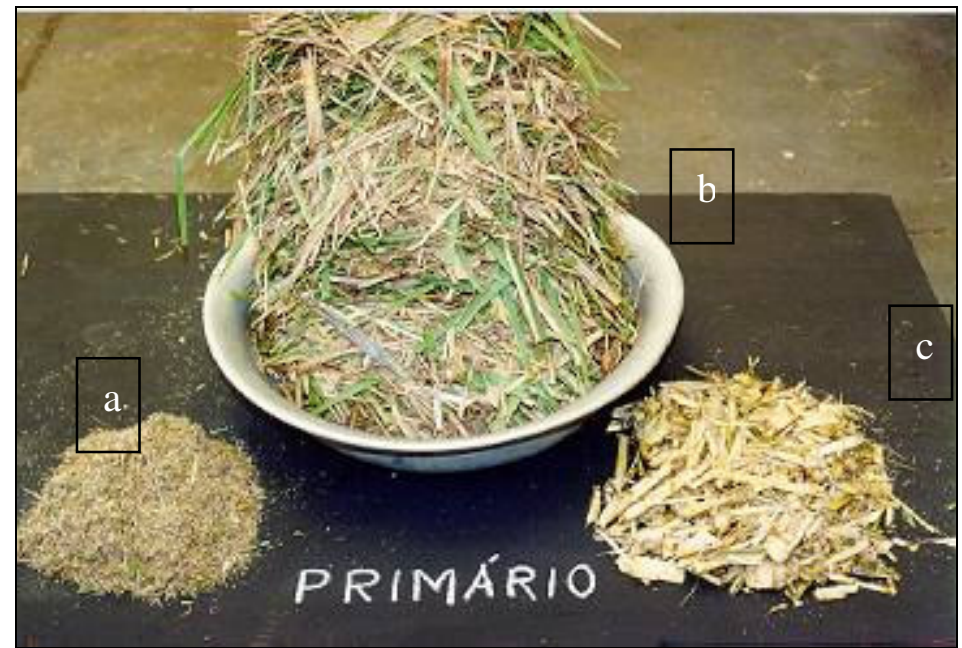

FIGURA 3. Perdas invisíveis, serragem (a) e lascas (c) recolhidas junto com as impurezas vegetais (b) na saída do extrator primário.

Pelos resultados obtidos, pode-se afirmar que o baixo desempenho das colhedoras, expresso pelos altos índices de perdas de matéria-prima, ou a baixa eficiência de limpeza não está associado à velocidade de deslocamento da colhedora ou ao potencial de produção do canavial. Esse desempenho está relacionado ao gradiente do fluxo de ar na câmara de limpeza, que apresenta grande variação na velocidade do ar, interferindo na capacidade de limpeza da colhedora e, por conseqüência, nas perdas totais, visíveis e invisíveis.

\section{CONCLUSÕES}

A velocidade de saída do ar dos extratores primário e secundário não é uniforme e apresenta alto coeficiente de variação; a velocidade média do ar de saída varia conforme a posição do elevador em relação à colhedora (direita ou esquerda), embora não apresente variação de sua distribuição em função de sua posição.

As perdas invisíveis nos sistemas das colhedoras foram significativas para as variedades utilizadas no ensaio e não apresentaram diferenças significativas quando se processaram diferentes fluxos de cana-de-açúcar.

A eficiência de limpeza da matéria-prima foi a mesma para as duas variedades de cana-deaçúcar analisadas, independentemente do fluxo de cana processada.

\section{AGRADECIMENTOS}

À Fundação de Amparo à Pesquisa do Estado de São Paulo (FAPESP) e ao Conselho Nacional de Desenvolvimento Científico e Tecnológico $(\mathrm{CNPq})$, pelo suporte financeiro. Aos pesquisadores, laboratoristas e técnicos do Centro Tecnológico Canavieiro (CTC), pelo suporte técnico.

\section{REFERÊNCIAS}

DICK, R.G.; RIDGE, D.R. The adoption of green cane harvesting and trash blanketing in Australia. In: INTERNATIONAL SOCIETY OF SUGAR CANE TECHNOLOGISTS CONGRESS, 20., 1989, São Paulo. Proceedings... São Paulo: The Organizing Committee of the ISSCT Congress, 1989. v.2, p.1034-42.

KROES, S.; HARRIS, H.D. Knockdown causes major damage to cane during harvesting. In: AUSTRALIAN SOCIETY OF SUGAR CANE TECHNOLOGISTS CONFERENCE, 18., 1996, Mackay. Proceedings... Brisbane: Watson Ferguson, 1996. p.137-44. 
MAGALHÃES, P.S.G.; BRAUNBECK, O.A. Colheita de cana-de-açúcar - atualidade e perspectiva. In: BAUBUENA R.H.; BENEZ, S.H.; JORIARURIA, D. Ingeniería Rural y Mecanización Agrária en el Ámbito Latinoamericano, La Plata, v.1, p.262-73, 1998.

MORAES, E.E. Avaliação das perdas invisíveis de cana-de-açúcar (Saccharum spp.) e impurezas vegetais na colheita mecanizada. 124 f. Dissertação (Mestrado em Máquinas Agrícolas) Faculdade de Engenharia Agrícola, UNICAMP, Campinas, 1992.

NEVES, J.L.M.; MAGALHÃES, P.S.G.; MORAES, E.E.; ARAUJO, F. Avaliação de perdas invisíveis de cana-de-açúcar nos sistemas da colhedora de cana picada. Engenharia Agrícola, Jaboticabal, v. 23, n. 3, p.539-46, 2003.

NEVES, J.L.M.; MAGALHÃES, P.S.G.; OTA, W.M. Sistema de monitoramento de perdas visíveis de cana-de-açúcar em colhedora de cana picada. Engenharia Agrícola, Jaboticabal, v.24, n.3, p.764-70, 2004.

RIP0LI, T.C.C; NERY, M.S.; MOLINA, W.F.; RIPOLI, M.L.C. Operational and economic performance of green cane chopped harvester, in Brazil. In: INTERNATIONAL ANNUAL MEETING ASAE, 2001, Sacramento. St. Joseph: ASAE, 2001a. Paper 011003.

RIP0LI, T.C.C; DE LEON, M.J.M.S.; MOLINA, W.F.; RIPOLI, M.L.C. Field testing of two green chopped sugar cane harvesters in Brazil. In: INTERNATIONAL ANNUAL MEETING ASAE, 2001, Sacramento. St. Joseph: ASAE, 2001b. Paper 011004. 